\title{
THE STRICT STABILITY OF DYNAMIC SYSTEMS ON TIME SCALES
}

\author{
S. SIVASUNDARAM \\ Embry-Riddle Aeronautical University \\ Department of Computing and Mathematics \\ Daytona Beach, FL 32114 USA
}

(Received March, 1999; Revised August, 2000)

\begin{abstract}
The strict stability of dynamic systems on time scales is examined with sufficient conditions. Results analogous to Lyapunov's theorems ae proved and discussed using a comparison principle.

Key words: Dynamic Systems, Time Scale, Strict Stability, Lyapunov Functions, Comparison Principles.
\end{abstract}

AMS subject classifications: 34D20, 39A11.

\section{Introduction}

Mathematical modeling of several important dynamic processes has been rendered via difference equations or differential equations. Difference equations also appear in the study of discretization methods for differential equations. From a modeling point of view, it is perhaps more realistic to model a phenomenon using a dynamic system that incorporates both continuous and discrete times, namely, time as an arbitrary closed set of reals called a time-scale. The recently developed dynamic systems on time scales off a unified approach to continuous and discrete systems [2].

The Lyapunov stability of the trivial solution of a differential system does not rule out the possibility of asymptotic stability. Moreover, the asymptotic stability of the trivial solution does not guarantee any information about the rate of decay of the solutions. Various definitions of stability are therefore one-sided estimates, and thus these are not strict concepts. It is natural to expect that an estimation of the lower bound for the rate at which solutions approach the trivial solution would offer interesting and useful refinements of the stability notions. Such concepts, known as stability in a tube-like domain, were introduced in [1].

Recently, in the development of the variational Lyapunov method [3], it has become necessary to employ the strict stability concept to prove a theorem analogous to Lyapunov's uniform asymptotic stability result. However, it was found that the earlier definitions of strict stability were too stringent for this purpose and that the ideas and proofs needed some further refinement. 
In this paper we discuss strict stability notions and give sufficient conditions for such concepts to hold. We first prove results analogous to Lyapunov's original theorems and then discuss them by employing a comparison principle.

\section{Preliminaries, Local Existence and Uniqueness}

Let $T$ be a time scale (closed nonempty subset of $R$ ) with $t_{0} \geq 0$ as a minimal element and no maximal element. The points $\{t\}$ of $\mathbb{T}$ are classified as

$$
\begin{aligned}
& \text { right-dense (rd), } \quad \text { if } \sigma(t)=t, \\
& \text { left-dense (ld), } \\
& \text { right-scattered (rs), } \rho(t)=t, \\
& \text { left-scattered (ls), } \sigma(t)>t,
\end{aligned}
$$

where $\sigma(t), \rho(t)$ are jump operators defined by

$$
\begin{aligned}
& \sigma(t)=\inf \{s \in \mathbb{T}: s>t\}, \\
& \rho(t)=\sup \{s \in \mathbb{T}: s<t\} .
\end{aligned}
$$

Set $m^{*}(t)=\sigma(t)-t($ called graininess $)$ so that

$$
\begin{gathered}
\mathbb{T} \equiv R \Rightarrow \mu^{*}(t)=0, \\
\mathbb{T} \equiv Z \Rightarrow \mu^{*}(t)=1 .
\end{gathered}
$$

Definition 2.1: The mapping $u: \mathbb{T} \rightarrow R$ is said to be $r d$-continuous if it is continuous at each right-dense point and $\lim _{s \rightarrow t}-f\left(t^{-}\right)$exists at each left-dense point.

Definition 2.2: A mapping $u: \mathbb{T} \rightarrow R$ is said to be differentiable at $t \in \mathbb{R}$, if there exist an $\alpha \in R$ such that for any $\epsilon>0$ there exists a neighborhood $U$ of $t$ satisfying

$$
|u(\sigma(t))-u(s)-\alpha(\sigma(t)-s)| \leq \epsilon|\sigma(t)-s| \text { for all } s \in U \text {. }
$$

Note: Derivative of $u$ is denoted by $u^{\Delta}(t)$, then

$$
\begin{gathered}
\mathbb{T}=R \Rightarrow u^{\Delta}=\alpha=\frac{d u(t)}{d t}, \\
\mathbb{T}=Z \Rightarrow u^{\Delta}=\alpha=u(t+1)-u(t) .
\end{gathered}
$$

If $u$ is differentiable at $t$, then it is continuous at $t$. If $u$ is continuous at $t$ and $t$ is right-scattered, then $u$ is differentiable and

$$
u^{\Delta}(t)=\frac{u(\sigma(t))-u(t)}{\mu^{*}(t)}
$$


Definition 2.3: For each $t \in \mathbb{R}$, let $N$ be a neighborhood of $t$. We define the generalized derivative (or Dini derivative), $D^{+} u^{\Delta}(t)$ as, given $\epsilon>0$, there exists a right neighborhood $N_{\epsilon} \subset N$ of $t$ such that

$$
\frac{u(\sigma(t))-u(s)}{\mu^{*}(t, s)}<D^{+} u^{\Delta}(t)+\epsilon \text { for } s \in N_{\epsilon}, s>t, \text { where } \mu(t, s)=\sigma(t)-s .
$$

In the case of $t$ being $\mathrm{rs}$ and $u$ continuous at $t$, we have, as in the case of the derivative,

$$
D^{+} u^{\Delta}(t)=\frac{u(\sigma(t))-u(t)}{\mu^{*}(t)} .
$$

Definition 2.4: Let $h$ be a mapping from $\mathbb{T}$ to $R$. The mapping $g: \mathbb{T} \rightarrow R$ is called the antiderivative of $h$ on $\mathbb{T}$ if it is differentiable on $\mathbb{T}$ and satisfies $g^{\Delta}(t)=h(t)$ for $t \in \mathbb{T}$.

The following known properties of the antiderivative are useful.

(a) If $h: \mathbb{T} \rightarrow R$ is rd-continuous, then $h$ has the antiderivative $g: t \rightarrow \int{ }_{s}^{t} h(s) d s$, $s, t \in \mathbb{T}$.

(b) If a sequence $\left\{h_{n}\right\}_{n \in N}$ of rd-continuous functions $\mathbb{T} \rightarrow R$ converges uniformly on $[r, s]$ to an rd-continuous function $h$, then $\left(\int{ }_{r}^{s} h_{n}(t) d t\right)_{n \in N} \rightarrow$ $\int{ }_{r}^{s} h(t) d t$, on $R$.

A basic tool employed in the proofs is the following induction principle, well suited for time scales. Suppose that for any $t \in \mathbb{T}$, there is a statement $A(t)$ such that the following conditions are verified:

(i) $\quad A\left(t_{0}\right)$ is true;

(ii) If $t$ is right-scattered and $A(t)$ is true, then $A(\sigma(t))$ is also true;

(iii) For each right-dense $t$, there exists a neighborhood $U$ such that whenever $A(t)$ is true, $A(s)$ is also true for all $s \in U, s \geq t$;

(iv) For left-dense $t, A(s)$ is true for all $s \in\left[t_{0}, t\right)$ and implies that $A(t)$ is true. Therefore, statement $A(t)$ is true for all $t \in \mathbb{T}$.

In the following we shall consider the initial value problem for dynamic systems on time scales and prove local existence and uniqueness results corresponding to Peano's and Perron's theorems. Let $\mathbb{T}^{k}$ represent the set of all nondegenerate points of the time scale $\mathbb{T}$. We consider the initial value problem (IVP)

$$
x^{\Delta}=f(t, x), t \in \mathbb{T}^{k}, x\left(t_{0}\right)=x_{0}
$$

where $f: \mathbb{T}^{k} \times R^{n} \rightarrow R^{n}$ and $f$ is rd-continuous on $\mathbb{T}^{k} \times R^{n}$. A map $x: \mathbb{T}^{k} \rightarrow R^{n}$ is a solution of IVP $(2.1)$ if $x(t)$ is an antiderivative of $f(t, x(t))$ on $\mathbb{T}^{k}$ and satisfies $x\left(t_{0}\right)=x_{0}$.

Theorem 2.1: Let $f \in C_{r d}\left[R_{0}, R^{n}\right]$ where $R_{0}=\left[t_{0}, t_{0}+a\right] \times B,\left[t_{0}, t_{0}+a\right]$ is understood as $\left[t_{0}, t_{0}+a\right] \cap \mathbb{T}^{k}$ and $B=\left\{x \in R^{n}:\left|x-x_{0}\right| \leq b\right\}$. Then, the IVP (2.1) has at least one solution $x(t)$ on $\left[t_{0}, t_{0}+\alpha\right]$, where $\alpha=\min \left(a, \frac{b}{M}\right)$ with $M$ being $a$ bound of $f(t, x)$ on $R_{0}$.

Proof: For any $r \in \mathbb{T}^{k}, t_{0}<r<t_{0}+\alpha$, define the mapping

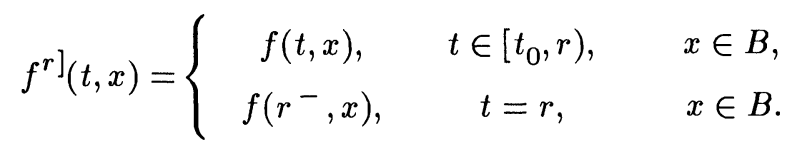


Let the statement $A(r)$ be as follows: The IVP

$$
x^{\Delta}=f^{r]}(t, x), t \in\left[t_{0}, r\right], x\left(t_{0}\right)=x_{0},
$$

has a solution $x_{r}(t)$ on $\left[t_{0}, r\right]$.

(i) The statement $A\left(t_{0}\right)$ is trivially true since the mapping

$$
x_{t_{0}}\left\{t_{0}\right\} \rightarrow B \text { and } x_{t_{0}}^{\Delta}(t)=f^{\left.t_{0}\right]}\left(t, x_{t_{0}}(t)\right) \text { for } t \in\left\{t_{0}\right\}^{k}=\emptyset .
$$

(ii) Let $r$ be right-scattered and $A(r)$ be true, i.e., IVP (2.1.r) has a solution $x_{r}(t)$ on $\left[t_{0}, r\right]$. Define the mapping

$$
x_{\sigma(r)}:\left[t_{0}, \sigma(r)\right\} \rightarrow B
$$

such that

$$
x_{\sigma(r)}(t)=\left\{\begin{array}{cc}
x_{r}(t), & t \in\left[t_{0}, r\right] \\
x_{r}(r)+f\left(r, x_{r}(r)\right) \mu^{*}(r), & t=\sigma(r) .
\end{array}\right.
$$

Then $x_{\sigma(r)}$ is continuous and is a solution of (2.1.r) on $\left[t_{0}, \sigma(r)\right]$.

(iii) ${ }^{\sigma}(r)$ Let $r$ be right-dense and $U_{r}$ be a neighborhood of $r$. Assume $A(r)$ is true. We need to prove that $A(s)$ is true for $s \in U_{r} \cap\left[t_{0}, t_{0}+\alpha\right], s>r$. By the classical existence theorem (Peano's theorem) there exists a solution $x_{s}(t)$ satisfying

$$
x_{s}^{\Delta}(t)=f\left(t, x_{s}(t)\right), t \in[r, s], s \in U_{r} \cap\left[t_{0}, t_{0}+\alpha\right]
$$

The mapping defined by

$$
x_{s}(r)=x_{r}(r)
$$

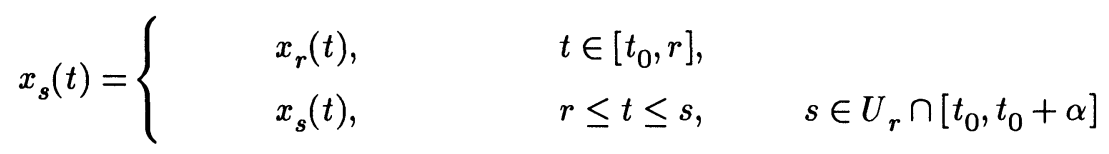

is a solution of (2.1.r) on $\left[t_{0}, s\right], s \geq r$, proving that $A(s)$ is true.

(iv) Let $r$ be left-dense such that $A()$ is true for all $s<r$. We need to prove that $A(r)$ is true. For any $s<r$, IVP $(2.1 . r)$ has a solution $x_{s}(t)$ on $\left[t_{0}, s\right]$ defined by

$$
x_{s}(r)=x_{0}+\int_{t_{0}}^{t} f\left(\tau, x_{s}(\tau)\right) \Delta \tau, t \in\left[t_{0}, s\right]
$$

Since $f(t, x)$ is rd-continuous, $\lim _{t \rightarrow r}-f\left(t, x_{s}(t)\right)$ exists and hence we have

$$
x_{s}(r)=x_{0}=\int_{t_{0}}^{r} f\left(\tau, x_{s}(\tau)\right) \Delta \tau
$$

Thus $x_{s}(t)$ is a solution of $(2.1 . r)$ on $\left[t_{0}, r\right]$, i.e., $A(r)$ holds.

By the induction principle, IVP $(2.1)$ has a solution on $\left[t_{0}, t_{0}+\alpha\right]$ and the proof is 
complete.

Next, we consider a Perron type uniqueness result.

Theorem 2.2: Assume that

(i) $g \in C_{r d}\left[\left[t_{0}, t_{0}+a\right] \times[0,2 b], R_{+}\right]$and for every $t_{1}, t_{0} \leq t_{1} \leq t_{0}+a, u(t) \equiv 0$ is the only solution of $u^{\Delta}=g(t, u), u\left(t_{1}\right)=0$, on $\left[t_{1}, t_{0}+a\right]$;

(ii) $f \in C_{r d}\left[R_{0}, R^{n}\right]$ and for each $t \in\left[t_{0}, t_{0}+a\right]$, there exists a compact neighborhood $U_{t}$ such that $f^{t]}$ in $U_{t} \times B$ satisfies

$$
|f(t, x)-f(t, y)| \leq g(t,|x-y|),(t, x),(t, y) \in U_{t} \times B .
$$

Then the IVP (2.1) has a unique solution $x(t)$ on $\left[t_{0}, t_{0}+a\right]$.

Proof: We apply the induction principle to the following statements

$A(r)$ : The IVP

$$
\left.x^{\Delta}=f^{r}\right](t, x), t \in\left[t_{0}, r\right], x\left(t_{0}=x_{0},\right.
$$

admits exactly one solution $x_{r}(\cdot)$.

(i) In fact, there exists only one mapping $x_{t_{0}}:\left\{t_{0}\right\} \rightarrow R^{n}$ with $x_{t_{0}}\left(t_{0}\right)=x_{0}$ and $x_{t_{0}}^{\Delta}(t)=f^{r]}\left(t, x_{t_{0}}(t)\right)$ for $t \in\left\{t_{0}\right\}^{k}=\emptyset$.

(ii) Let $r$ be right-scattered. IVP (2.2.r) has, according to the induction condition, exactly one solution $x_{r}(\cdot)$. We define mapping $x_{\sigma(r)}:\left[t_{0}, \sigma(r)\right] \rightarrow R^{n}$ by

$$
x_{\sigma(r)}(t)=\left\{\begin{array}{cc}
x_{r}(t), & \text { if } t \in\left[t_{0}, r\right], \\
x_{r}(r)+f\left(r, x_{r}(r)\right) \mu^{*}(r), & \text { if } t=\sigma(r) .
\end{array}\right.
$$

It is continuous and the only solution of IVP $(2.2 . \sigma(r))$, since its restriction to $\left[t_{0}, r\right]$ is the only solution of the IVP $(2.2 . r)$ and its restriction to $[r, \sigma(r)]$ is the only solution of the IVP

$$
x^{\Delta}=f(t, x), x(r)=x_{r}(r) \text { on }[r, \sigma(r)] .
$$

(iii) Let $r$ be right-dense. By the induction condition, there exists exactly one solution $x_{r}(\cdot)$ of (2.2.r). Let $V_{r} \subseteq U_{r}$ be a compact neighborhood of $r$. By Perron's Theorem, for each $s \in V_{r}, s \geq t$ the IVP

$$
x^{\Delta}=f^{s]}(t, x), t \in[r, s], x(r)=x_{r}(r)
$$

admits exactly one solution $y_{s}(\cdot)$. The mapping $x_{s}$, defined by

$$
x_{s}(t):= \begin{cases}x_{r}(t), & \text { if } t \in\left[t_{0}, r\right] \\ y_{s}(t), & \text { if } t \in[r, s]\end{cases}
$$

is the unique solution of the IVP (2.2.s). Hence we have $A(s)$ for all $s \in V_{r}, s \geq r$.

(iv) Let $r$ be left-dense, and choose $V_{r}$ as above, then there is a $s \in V_{r}$ with $s<r$. With the help of the induction condition $A(s)$ and Perron's Theorem, the existence and uniqueness of a solution $x_{r}(\cdot)$ of $(2.2 . r)$ can be shown exactly in the same way as in (iii). Hence we have $A(r)$.

Since there is a unique solution on each interval $\left[t_{0}, r\right], r \geq t_{0}$, there is a unique solution of $(2.1)$ on $\left[t_{0}, t_{0}+a\right]$. Thus the proof is complete. 
Let $\mathscr{K}=\left\{a \in C_{r d}\left[\mathbb{T}, R_{+}\right]: a(u)\right.$ is strictly increasing in $u, a(0)=0$ and $a(u) \rightarrow \infty$ as $u \rightarrow \infty\}$ and consider the initial value problem (IVP)

$$
x^{\Delta}=f(t, x), t \in \mathbb{R}, x\left(t_{0}\right)=x_{0},
$$

where $f: \mathbb{T} \times R^{n} \rightarrow R^{n}$ and $f$ is rd-continuous on $\mathbb{T} \times R^{n}$.

Definition 2.5: The trivial solution of (2.1) is said to be:

(S1) strictly stable, if given $\epsilon_{1}>0$ and $t_{0} \in \mathbb{T}$, there exists a $\delta=\delta_{1}\left(t_{0}, \epsilon_{1}\right)>0$ such that $\left|x_{0}\right|<\delta_{1}$ implies $|x(t)|<\epsilon_{1}, t \geq t_{0}$, and for every $0<\delta_{2} \leq \delta_{1}$, there exists a

$$
0<\epsilon_{2}<\delta_{2} \text { such that } \delta_{2}<\left|x_{0}\right| \text { implies } \epsilon_{2}<|x(t)|, t \geq t_{0}
$$

(S2) strictly uniformly stable, if $\delta_{1}, \delta_{2}$ and $\epsilon_{2}$ are independent of $t_{0}$;

(S3) strictly attractive, if given $\alpha_{1}>0, \epsilon_{1}>0$ and $t_{0} \in \mathbb{T}$ for every $\alpha_{2} \leq \alpha_{1}$ there exists $\epsilon_{2}<\epsilon_{1}, T_{1}=T_{1}\left(t_{0}, \epsilon_{1}\right)$, and $T_{2}=T_{2}\left(t_{0}, \epsilon_{1}\right)$ such that

$\alpha_{2} \leq\left|x_{0}\right| \leq \alpha_{1}$ implies $\epsilon_{2}<|x(t)|<\epsilon_{1}$, for $t_{0}+T_{1} \leq t \leq t_{0}+T_{2}$;

(S4) strictly uniformly attractive if $T_{1}$ and $T_{2}$ in (S3) are independent of $t_{0}$;

(S5) strictly asymptotically stable if (S3) holds and the trivial solution is stable;

(S6) strictly uniformly asymptotically stable if (S4) holds and the trivial solution is uniformly stable.

Remark 2.1: It is important to note that (S1) and (S3), or, (S2) and (S4) cannot hold at the same time. If in (S1) it is not possible to find an $\epsilon_{2}$ satisfying (2.2), we shall say that the trivial solution is stable. This can happen when $|x(t)| \rightarrow 0$ as $t \rightarrow \infty$, or, $\lim \inf |x(\mathrm{t})|=0$ and $\lim \sup |x(t)| \neq 0$.

\section{Main Results}

In this section we discuss sufficient conditions for the strict stability notions.

Theorem 3.1: Assume that

$\left(H_{1}\right)$ for each $0<\eta<\rho, V_{e} \in C_{r d}\left[\mathbb{\top} \times S_{\rho}, R_{+}\right], V_{\eta}$ is locally Lipschitzian in $x$ and for $(t, x) \in \mathbb{T} \times S_{\rho}$ and $|x| \geq \eta$,

and

$$
b_{1}(|x|) \leq V_{\eta}(t, x) \leq a_{1}(|x|), a_{1}, b_{1} \in K
$$

$$
D^{+} V_{\eta}^{\Delta}(t, x) \leq 0
$$

$\left(H_{2}\right)$ for each $\sigma, 0<\sigma<\rho, V_{\sigma} \in C_{r d}\left[\mathbb{T} \times e S_{\rho}, R_{+}\right], V_{\sigma}$ is locally Lipschitzian in $x$ and for $(t, x) \in \mathbb{T} \times S_{\rho}$ and $|x| \leq \sigma$,

and

$$
b_{2}(|x|) \leq V_{\eta}(t, x) \leq a_{2}(|x|), a_{2}, b_{2} \in K
$$

$$
D^{+} V_{\eta}^{\Delta}(t, x) \geq 0 \text {. }
$$

Then the trivial solution is strictly uniformly stable.

Proof: Let $0<\epsilon<\rho$ and $t_{0} \in \mathbb{T}$ be given. Choose $\delta_{1}=\delta_{1}\left(\epsilon_{1}\right)>0$ such that

$$
a_{1}\left(\delta_{1}\right)<b_{1}\left(\epsilon_{1}\right)
$$


Then we claim that

$$
\left|x_{0}\right|<\delta_{1} \text { implies }|x(t)|<\epsilon_{1}, t \geq t_{0} .
$$

If (3.3) is not true, then there would exist $t_{1}, t_{2} \in \mathbb{T}, t_{1}>t_{2}>t_{0}$ and a solution of (2.1) with $\left|x_{0}\right|<\delta_{1}$, satisfying $\left|x\left(t_{1}\right)\right|=\epsilon_{1},\left|x\left(t_{2}\right)\right|=\delta_{1}$ and $\delta_{1} \leq|x(t)|$ for $t \in\left[t_{2}, t_{1}\right]$.

Choosing $\eta=\delta_{1}$, and using $\left(H_{1}\right)$, we obtain

$$
b_{1}\left(\epsilon_{1}\right)=b_{1}\left(\left|x\left(t_{1}\right)\right|\right) \leq V_{\eta}\left(t_{1}, x\left(t_{1}\right)\right) \leq V_{\eta}\left(t_{2}, x\left(t_{2}\right)\right) \leq a_{1}\left(\left|x\left(t_{2}\right)\right|\right)=a_{1}\left(\delta_{1}\right),
$$

which contradicts (3.3). Hence (3.4) is valid.

Now let $0<\delta_{2} \leq \delta_{1}$ and choose $0<\left|x_{0}\right|<\delta_{2}<\delta_{1}$ such that

We now claim that

$$
a_{2}\left(\epsilon_{2}\right)<b_{2}\left(\delta_{2}\right)
$$

$$
\delta_{2}<\left|x_{0}\right|<\delta_{1} \text { implies } \epsilon_{2}<|x(t)|<\epsilon_{1}, t=t_{0} .
$$

If (3.6) is false, then because of (3.4), there exists a solution of (2.1) with $\delta_{2}<$ $\left|x_{0}\right|<\delta_{1}$ and $t_{1}>t_{2}>t_{0}$ satisfying

$$
\left|x\left(t_{1}\right)\right|=\epsilon_{2},\left|x\left(t_{2}\right)\right|+\delta_{2} \text { and }|x(t)| \leq \delta_{2} \text { for } t \in\left[t_{2}, t_{1}\right]
$$

Let $\sigma=\delta_{2}$ and using $\left(H_{2}\right)$, we get

$$
a_{2}\left(\epsilon_{2}\right)=a_{2}\left(\left|x\left(t_{1}\right)\right|\right) \geq V_{\sigma}\left(t_{1}, x\left(t_{1}\right)\right) \geq V_{\sigma}\left(t_{2}, x\left(t_{2}\right)\right) \geq b_{2}\left(\left|x\left(t_{2}\right)\right|\right)
$$

which contradicts (3.5). Thus (3.6) is valid and hence uniform strict stability of the trivial solution of (2.1) follows. This completes the proof of Theorem 3.1.

Theorem 3.2: Let the assumptions of Theorem 3.1 hold, except that the conditions (3.1) and (3.2) are replaced by

and

$$
D^{+} V_{\eta}^{\Delta}(t, x) \leq-c_{1}(|x|)
$$

$$
D^{+} V_{\eta}^{\Delta}(t, x) \geq-c_{2}(|x|)
$$

where $c_{1}, c_{2} \in K$. Then the trivial solution of (2.1) is strictly uniformly asymptotically stable.

Proof: First we note that although (3.8) implies (3.1), (3.9) does not yield (3.2). As a result, we obtain because of (3.8) only uniform stability of the trivial solution of $(2.1)$, i.e.,

$$
\left|x_{0}\right|<\delta_{1} \text { implies }|x(t)|<\epsilon_{1}, t \geq t_{0}, t \in \mathbb{T} \text {. }
$$

Now, to prove the conclusion of Theorem 3.2, we need to show that the trivial solution of (2.1) is strictly uniformly attractive. For this purpose, we let $\epsilon_{1}=\rho$ and designate by $\delta_{10}=\delta_{1}(\rho)$ so that $(3.10)$ yields

$$
\left|x_{0}\right|<\delta_{10} \text { implies }|x(t)|<\rho, t \geq t_{0}, t \in \mathbb{T} \text {. }
$$

Let $\left|x_{0}\right|<\delta_{10}$. We show, using standard argument, that there exists a $t^{*} \in$ $\left[t_{0}, t_{0}+T\right]$, where $T=T(\epsilon)>\frac{a_{1}\left(\delta_{10}\right)}{c_{1}\left(\delta_{1}\right)}$ with $\delta_{1}$ is the number corresponding to $\epsilon_{1}$ in uniform stability, such that $\left|x\left(t^{*}\right)\right|<\delta_{1}$ for any solution $x(t)$ of (2.1) with $\left|x_{0}\right|<\delta_{10}$. If this is not true we will have $|x(t)| \geq \delta_{1}, t \in\left[t_{0}, t_{0}+T\right]$. Then, letting $\eta=\delta_{1}$ and using $\left(H_{1}\right)$ with (3.8), we have 


$$
\begin{gathered}
b_{1}(\delta)=b_{1}\left(\left|x\left(t_{0}+T\right)\right|\right) \leq V_{\eta}\left(t_{0}+T, x\left(t_{0}+T\right)\right. \\
\leq V_{\eta}\left(t_{0}, x_{0}\right)=\int_{t_{0}}^{t_{0}+T} c_{1}(|x|) \Delta s<a_{1}\left(\delta_{10}\right)-c_{1}\left(\delta_{1}\right) T=b_{1}\left(\delta_{1}\right),
\end{gathered}
$$

in view of the choice of $T$. This contradiction implies that there exists a $t^{*} \in$ $\left[t_{0}, t_{0}+T\right]$, satisfying $\left|x\left(t^{*}\right)\right|<\delta_{1}$. Due to the uniform stability of the trivial solution of (2.1), this yields that $|x(t)|<\epsilon_{1}, t \geq t_{0}+T \geq t^{*}$, which implies that there exists a $t_{0}<T_{1}<T$ such that $\left|x\left(t_{0}+T\right)\right|=\epsilon_{1}$. Now for any $\delta_{20}, 0<\delta_{20}<\delta_{10}$, choose $\epsilon_{2}$ such that $b_{2}\left(\delta_{20}\right)>a_{2}\left(\epsilon_{2}\right)$ and $0<\epsilon_{2}<\epsilon_{1}<\delta_{20}$. Suppose that $\delta_{20}<$ $\left|x_{0}\right|<\delta_{10}$. Let us define

$$
\tau=\frac{\left[b_{2}\left(\delta_{20}\right)-a_{2}\left(\epsilon_{2}\right)\right]}{c_{2}\left(\epsilon_{1}\right)}, \text { and } T_{2}=T_{1}+\tau .
$$

Since $|x(t)| \leq \epsilon_{1}$, for $t \geq t_{0}+T_{1}$, choosing $\sigma=\epsilon_{1}$ and using $\left(H_{2}\right)$ with (3.9), we get for $t \in\left[t_{0}+T_{1}, t_{0}+T_{2}\right]$,

$$
\begin{gathered}
a_{2}(|x(t)|) \geq V_{\sigma}(t, x(t)) \\
\geq V_{\sigma}(t, x(t))-\int_{t_{0}}^{t} c_{2}(|x(s)|) \Delta s \geq b_{2}\left(\delta_{20}\right)-\int_{t_{0}+T_{1}}^{t} c_{2}(|x(s)|) \Delta s \\
\geq b_{2}\left(\delta_{20}\right)-c_{2}\left(\epsilon_{1}\right)\left[t-\left(t_{0}+T_{1}\right)\right] .
\end{gathered}
$$

Since $t-\left(t_{0}+T_{1}\right) \geq \tau$, it follows that

$$
a_{2}(|x(t)|) \geq b_{2}\left(\delta_{20}\right)-c_{2}\left(\epsilon_{1}\right) \frac{\left[b_{2}\left(\delta_{20}\right)-a_{2}\left(\epsilon_{2}\right)\right]}{c_{2}\left(\epsilon_{1}\right)}=a_{2}\left(\epsilon_{2}\right) .
$$

This yields that

and therefore,

$$
|x(t)| \geq \epsilon_{2}, \text { for } t \in\left[t_{0}+T_{1}, t_{0}+T_{2}\right],
$$

$$
\epsilon_{2}<|x(t)|<\epsilon_{1} \text { for } t \in\left[t_{0}+T_{1}, t_{0}+T_{2}\right],
$$

which completes the proof.

Before proving the general result in terms of the comparison principle, we need to consider the comparison differential system

$$
\left\{\begin{array}{l}
u_{1}^{\Delta}=g_{1}\left(t, u_{1}\right), u_{1}\left(t_{0}\right)=u_{0} \geq 0 \\
u_{2}^{\Delta}=g_{2}\left(t, u_{2}\right), u_{2}\left(t_{0}=u_{0} \geq 0\right.
\end{array}\right.
$$

where $g_{1}, g_{2} \in C_{r d}\left[\mathbb{T} \times R_{+}, R\right]$. We shall say that the comparison system (3.12) is strictly stable, if given $\epsilon_{1}>0$ and $t_{0} \in \mathbb{T}$, there exists a $\delta_{1}>0$ such that $u_{0}<\delta_{1}$ implies $u_{1}(t)<\epsilon_{1}, t \geq t_{0}$, and for every $\delta_{2} \leq \delta_{1}$, there exists an $\epsilon_{2}, 0<\epsilon_{2}<\delta_{2}$ such that $\delta_{2}<u_{0}$ implies that $\epsilon_{2}<u_{2}(t), t \geq t_{0}$. Here, $u_{1}(t)$ and $u_{2}(t)$ are any solutions of $(3.12 a)$ and $(3.12 b)$, respectively.

Based on these definitions, we can formulate other strict stability notions. The next result is formulated in terms of comparison principles. 
Theorem 3.3: $\quad$ Let the assumptions of Theorem 3.1 hold, except that conditions (3.1) and (3.2) are replaced by

and

$$
D^{+} V_{\eta}^{\Delta}(t, x) \leq g_{1}\left(t, v_{\eta}(t, x)\right),(t, x) \in \mathbb{T} \times R^{n}
$$

$$
D^{+} V_{\sigma}^{\Delta}(t, x) \geq g_{2}\left(t, V_{\sigma}(t, x)\right),(t, x) \in \mathbb{T} \times R^{n}
$$

where $g_{2}(t, u) \leq g_{1}(t, u), g_{1}, g_{2} \in C_{r d}\left[\mathbb{T} \times R_{+}, R\right], g_{1}(t, 0) \equiv 0, g_{2}(t, 0) \equiv 0$. Then any strict stability concept of the comparison system implies the corresponding strict stability concept of the trivial solution of (2.1), respectively.

Proof: $\mathrm{L}$ et $0<\epsilon_{1}<\rho$ and $t_{0} \in \mathbb{T}$ be given. Suppose that the trivial solution of the comparison system (3.12) is strictly uniformly stable. Then for any given $b_{1}\left(\epsilon_{1}\right)>0$ and $t_{0} \in \mathbb{T}$, there exists $\delta_{1}^{*}>0$ such that

$$
0<u_{0}<\delta_{1}^{*} \text { implies that } u_{1}(t)<b_{1}\left(\epsilon_{1}\right), t \geq t_{0},
$$

where $u_{1}(t)=u_{1}\left(t, t_{0}, u_{0}\right)$ is any solution of $(3.12 a)$. Choose $\delta_{1}>0$ such that $a_{1}\left(\delta_{1}\right) \leq \delta_{1}^{*}$. Then we claim that

$$
\left|x_{0}\right|<\delta_{1} \text { implies }|x(t)|<\epsilon_{1}, t \geq t_{0} \text {. }
$$

If (3.15) is not true, then there exist $t_{1}, t_{2}, t_{1}>t_{2}>t_{0}$ and a solution of (2.1) with $\left|x\left(t_{2}\right)\right|=\delta_{1}, \quad\left|x\left(t_{1}\right)\right|=\epsilon_{1}$ and $\delta_{1}<|x(t)|<\epsilon_{1}$, for $t \in\left[t_{2}, t_{1}\right]$. Choosing $\eta=\delta_{1}$ and using the theory of differential inequalities, together with $\left(H_{1}\right)$, we get, by $(3.13)$ and (3.15),

$$
\begin{gathered}
b_{1}\left(\epsilon_{1}\right) \leq b_{1}\left(\left|x\left(t_{1}\right)\right|\right) \leq V_{\eta}\left(t_{1}, x\left(t_{1}\right)\right) \leq r\left(t_{1}, t_{2}, V_{\eta}\left(t_{2}, x\left(t_{2}\right)\right)\right. \\
\leq r\left(t_{1}, t_{2}, a_{1}\left(\delta_{1}\right)\right) \leq r\left(t_{1}, t_{2}, \delta^{*}\right)<b_{1}\left(\epsilon_{1}\right),
\end{gathered}
$$

which is a contradiction. Here $r\left(t, t_{0}, u_{0}\right)$ is the maximal solution of $(3.12 a)$. Hence, (3.16) is true.

Now, by strict uniform stability of the comparison system, we also have, for any $\delta_{2}^{*} \leq \delta_{1}^{*}$, there exists an $\epsilon_{2}^{*}<\delta_{2}^{*}$ satisfying

$$
\delta_{2}^{*}<u_{0} \text { implies } u_{2}(t)>\epsilon_{2}^{*}, t \geq t_{0}
$$

For any $\delta_{2} \leq \delta_{1}$, with $b_{2}\left(\delta_{2}\right) \geq \delta_{2}^{*}$, choose $\epsilon_{2}<\delta_{2}$ such that $\epsilon_{2}^{*} \geq a_{2}\left(\epsilon_{2}\right)$. By following an argument similar to the one used to establish (3.4) in Theorem 3.1, we can conclude that $\delta_{2}<\left|x_{0}\right|$ implies that $\epsilon_{2}<|x(t)|$ for $t>t_{0}$. Let $\sigma=\delta_{2}$. Then using the theory of differential inequalities, $(3.12 b),\left(H_{2}\right)$ and $(3.17)$, it follows that

$$
\begin{gathered}
a_{2}\left(\epsilon_{2}\right) \geq a_{2}(|x(t)|) \geq V_{\sigma}\left(t_{2}, x\left(t_{2}\right)\right) \\
\geq \rho\left(t_{1}, t_{2}, V_{\sigma}\left(t_{2}, x\left(t_{2}\right)\right)\right. \\
\geq \rho\left(t_{1}, t_{2}, b_{2}\left(\delta_{2}\right)\right) \geq \rho\left(t_{1}, t_{2}, \delta_{2}^{*}\right)>\epsilon_{2}^{*} \geq a_{2}\left(\epsilon_{2}\right)
\end{gathered}
$$

where $\rho\left(t, t_{0}, u_{0}\right)$ is the minimal solution of $(3.12 b)$. This is a contradiction and consequently, the trivial solution of $(2.1)$ is strictly uniformly stable.

Next, assume that the trivial solution of the comparison system (3.12) is strictly uniformly asymptotically stable. We see that the trivial solution of $(3.12 a)$ is uniformly stable. That is, 


$$
\left|x_{0}\right|<\delta_{1} \text { implies }|x(t)| \leq \epsilon_{1}, t \geq t_{0}
$$

To complete the proof, we need to prove that the trivial solution of $(2.1)$ is strictly uniformly attractive. To show this, fix $\epsilon_{1}=\rho$ and designate $\delta_{10}=\delta_{1}(\rho)$, so that we have

$$
\left|x_{0}\right|<\delta_{10} \text { implies }|x(t)|<\rho, t \geq t_{0}
$$

Let $\left|x_{0}\right|<\delta_{10}$. Let $\epsilon_{1}>0$ and $t_{0} \in \mathbb{T}$ be given. Choose $\alpha_{1}=\delta_{10}$. Let $\epsilon_{1}^{*} \leq b_{1}\left(\epsilon_{1}\right)$ and $\alpha_{1}^{*} \geq a_{1}\left(\delta_{10}\right)$. For any $\alpha_{2}=\delta_{20}<\delta_{10}$, define $\alpha_{2}^{*} \leq b_{2}\left(\delta_{20}\right)$ and $\epsilon_{2}^{*} \geq a_{2}\left(\epsilon_{2}\right)$ for any $\epsilon_{2}^{*}<\epsilon_{1}^{*}$. Assume that the comparison system is strictly uniformly asymptotically stable. Since this implies strict uniform attractivity, given $\alpha_{1}^{*}>0, \epsilon_{1}^{*}>0$ and $t_{0} \in \mathbb{T}$, for every $\alpha_{2}^{*} \leq \alpha_{1}^{*}$, there exist $\epsilon_{2}^{*}<\epsilon_{1}^{*}$ and $T_{1}<T_{2}$ such that $\alpha_{2}^{*} \leq u_{0} \leq \alpha_{1}^{*}$ implies that

$$
\epsilon_{2}^{*}<u_{2}(t) \leq u_{1}(t)<\epsilon_{1}^{*}, n\left[t_{0}+T_{1}, t_{0}+T_{2}\right]
$$

Take any $\delta_{20}<\delta_{10}$ and let $\delta_{20}<\left|x_{0}\right|<\delta_{10}$. Then using $\left(H_{1}\right),(3.15)$ and $(3.12 a)$, we get for $t \in\left[t_{0}+T_{1}, t_{0}+T_{2}\right]$,

$$
\begin{gathered}
\left.b(|x(t)|) \leq V_{\eta}(t)\right) \leq r\left(t, t_{0}, V_{t}\left(t_{0}, x\left(t_{0}\right)\right)\right. \\
\leq r\left(t_{1}, t_{2}, a_{1}\left(\left|x_{0}\right|\right)\right) \leq r\left(t_{1}, t_{2}, \delta_{10}^{*}\right)<\epsilon_{1}^{*}<b_{1}\left(\epsilon_{1}\right),
\end{gathered}
$$

which implies that $|x(t)|<\epsilon_{1}, t \in\left[t_{0}+T_{1}, t_{0}+T_{2}\right]$. Similarly, using $\left(H_{2}\right),(3.17)$ and $(3.12 b)$, we see that for $t \in\left[t_{0}+T_{1}, t_{0}+T_{2}\right]$,

$$
\begin{aligned}
& a_{2}(|x(t)|) \geq V_{\sigma}(t, x(t)) \geq \rho\left(t, t_{0}, V_{\sigma}\left(t_{0}, x\left(t_{0}\right)\right)\right. \\
& \geq \rho\left(t, t_{0}, b_{2}\left(\left|x_{0}\right|\right)\right) \geq \rho\left(t, t_{0}, \delta_{20}\right)>\epsilon_{2}^{*} \geq a_{2}\left(\epsilon_{2}\right),
\end{aligned}
$$

which yields that $x(t) \mid>\epsilon_{2}, t_{0}+T_{1}<t<t_{0}+T_{2}$. Thus (3.19) and (3.20) yield that $\epsilon_{2}<|x(t)|<\epsilon_{1}$, for $t \in\left[t_{0}+T_{1}, t_{0}+T_{2}\right]$ whenever $\delta_{20}<\left|x_{0}\right|<\delta_{10}$. Thus the proof is complete.

\section{References}

[1] Lakshmikantham, V. and Leela, S., Differential and Integral Inequalities Vol. 1, Academic Press, New York 1969.

[2] Lakshmikantham, V., Sivasundaram, S. and Kaymakcalan, B., Dynamic Systems on Measure Chains, Kluwer Academic Publishers, The Netherlands 1996.

[3] Rajalakshmi, S. and Sivasundaram, S., Variational Lyapunov second method, Dyn. Sys. and Appl. 2 (1993), 485-490. 


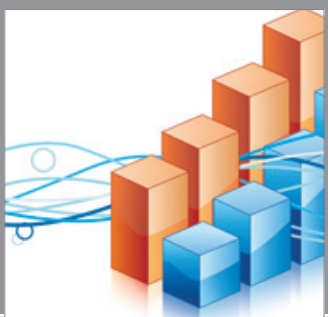

Advances in

Operations Research

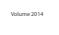

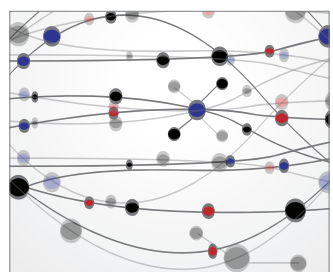

\section{The Scientific} World Journal
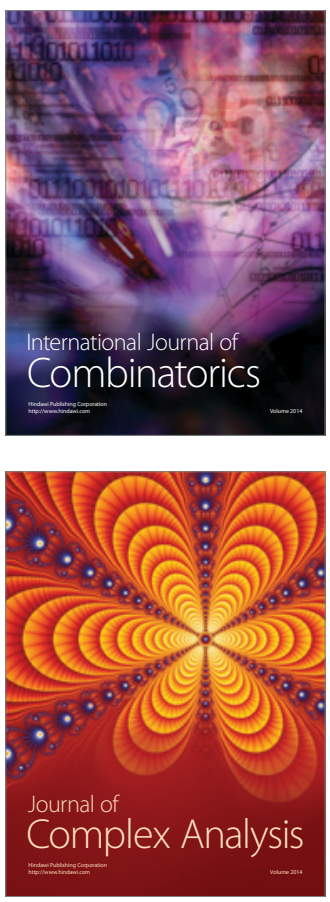

International Journal of

Mathematics and

Mathematical

Sciences
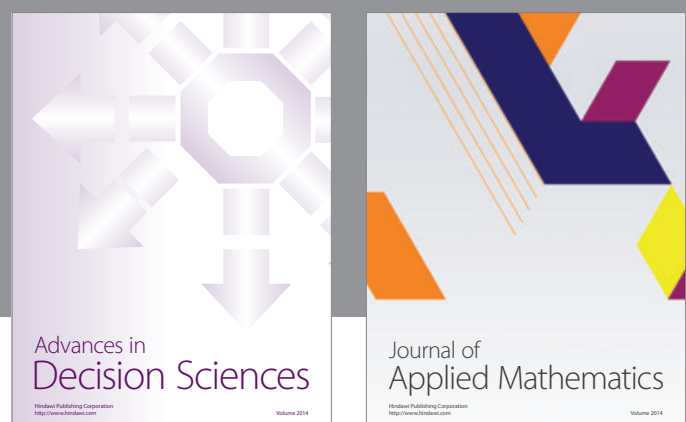

Journal of

Applied Mathematics
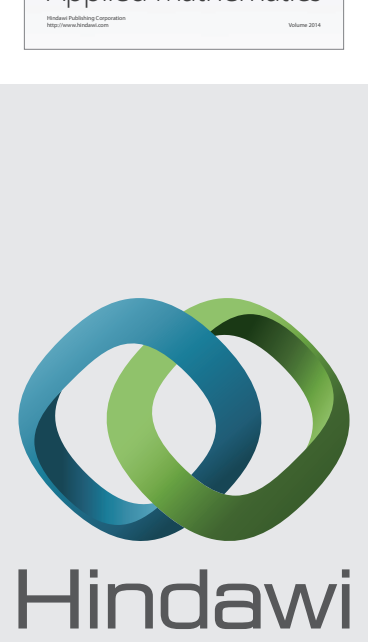

Submit your manuscripts at http://www.hindawi.com
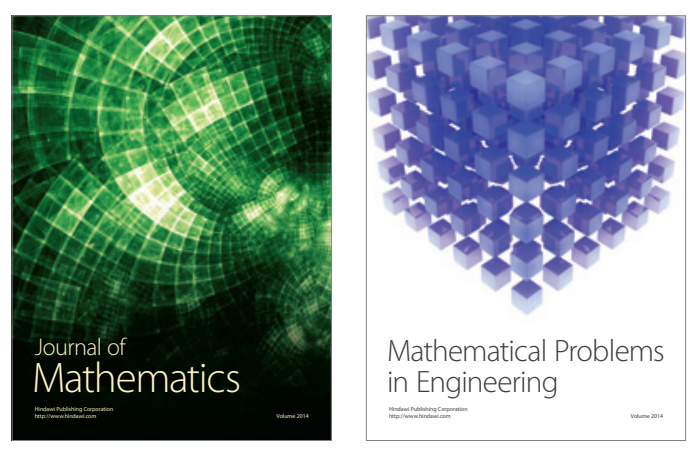

Mathematical Problems in Engineering
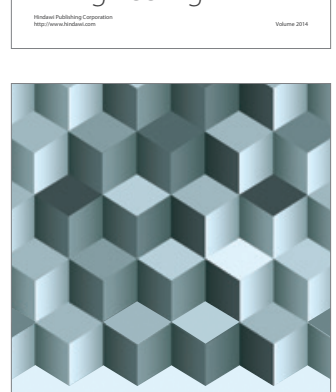

Journal of

Function Spaces
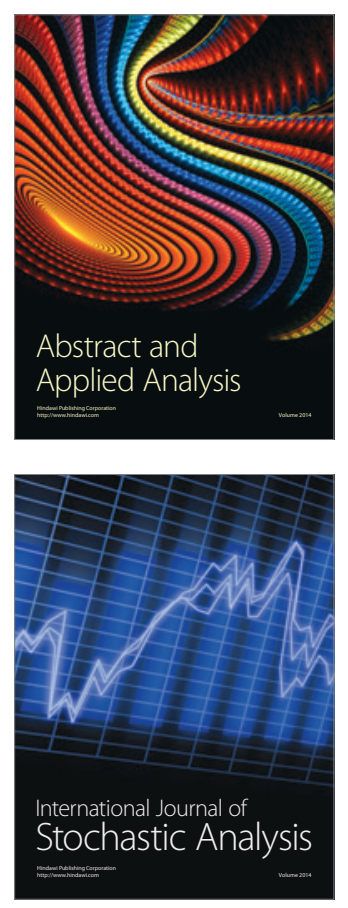

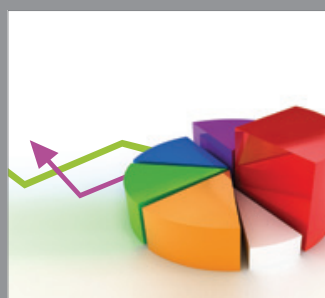

ournal of

Probability and Statistics

Promensencen
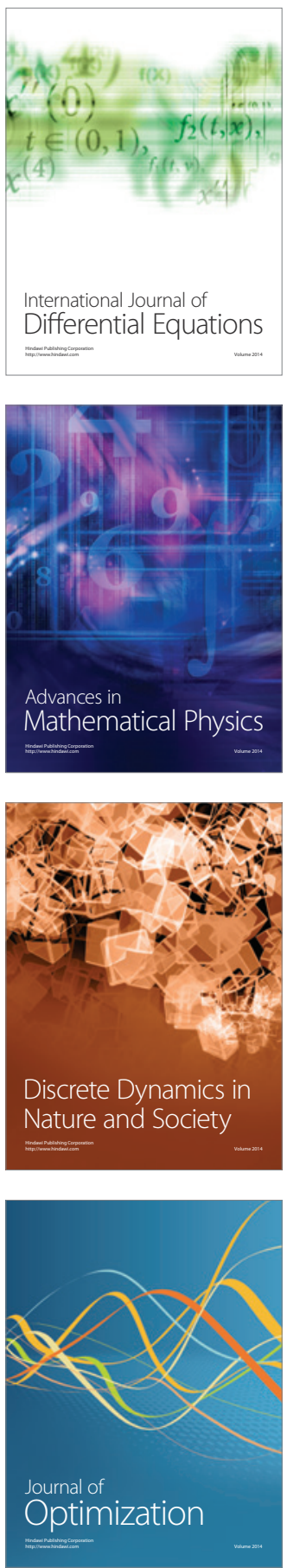